\title{
The Ability to Visually Recognize Facial Emotions during the Initial Stages of Alzheimer's
}

\section{Philippe Granato ${ }^{*}$, Shreekumar Vinekar ${ }^{2}$, Olivier Godefroy ${ }^{3}$, Jean-Pierre Van Gansberghe ${ }^{4}$, Raymond Bruyer ${ }^{5}$}

${ }^{1}$ Service de Psychiatrie, Centre Hospitalier de Denain, Denain, France

${ }^{2}$ Department of Psychiatry and Behavioral Sciences, University of Oklahoma College of Medicine, Oklahoma City, OK, USA

${ }^{3}$ Centre Hospitalier Universitaire d'Amiens, Neurologie, Amiens, France

${ }^{4}$ Data Processing Consultant in Mathematics, Brussels, Belgium (Died)

${ }^{5}$ Unité de Neurosciences Cognitives (NESC), Louvain-la-Neuve, Belgium

Email: *philippe.granato@gmail.com

How to cite this paper: Granato, P., Vinekar, S., Godefroy, O., Van Gansberghe, J.-P. and Bruyer, R. (2020) The Ability to Visually Recognize Facial Emotions during the Initial Stages of Alzheimer's. Open Journal of Psychiatry, 10, 187-204.

https://doi.org/10.4236/ojpsych.2020.104016

Received: January 1, 2020

Accepted: September 21, 2020

Published: September 24, 2020

Copyright $\odot 2020$ by author(s) and Scientific Research Publishing Inc. This work is licensed under the Creative Commons Attribution International License (CC BY 4.0).

http://creativecommons.org/licenses/by/4.0/

\begin{abstract}
Background: Alzheimer's sufferers (AS) are unable to visually recognize facial emotions (VRFE). However, we do not know the kind of emotions involved, the timeline for the onset of this loss of ability to recognize facial emotional expressions during the natural course of this disease and the existence of any correlation with other comorbid cognitive disorders. For that reason, the authors aimed to determine whether a deficit in facial emotion recognition is present at the onset of Alzheimer disease, distinctly and concurrently with the onset of cognitive impairment or is it a prodromal syndrome of Alzheimer's Disease before the onset of cognitive decline and what emotions are involved. A secondary aim was to investigate relationships between facial emotion recognition and cognitive performance on various parameters. Method: Single Blind Case-control study. Setting in Memory clinic. Participants: 12 patients, (AS) and 12 control subjects (CS) were enrolled. Measurements: Quantitative information about the ability for facial emotion recognition was obtained from Method of Analysis and Research on the Integration of Emotions (MARIE). The Mini Mental Status Examination (MMSE), the Picture Naming, the Mattis Dementia Rating Scale (DRS), and the Grober \& Buschke Free and Cued Selective Reminding Test (FCSRT) tests were used to measure cognitive impairment. Results: We note that the AS have a problem with the visual recognition of facial emotions with existence of a higher threshold for visual recognition. The AS is less sensitive to the visual recognition cues of facial emotions. AS is unable to distinguish anger from fear. It would be a possi-
\end{abstract}


ble explanation for some acts of aggressiveness seen in the clinical and home setting demonstrated by " $A S$ with behavioral disturbance". The anger-fear series was found to be the first affected in the course of Alzheimer's. The appearance of the curve is sigmoid for the control group and linear for the Alzheimer's patients with a cognitive distortion when the VRFE is represented graphically with percentage of correct recognition plotted on the " $y$ " axis and the selected images presented as stimulus with measures of density of emotion plotted on the " $x$ " axis. In both groups, it is intuitively and theoretically expected that correct recognition will be directly proportional to the density of represented emotion in the stimulus image. This hypothesis is true for CS but not so for AS. The MARIE (see below) processing of emotions seems to be strengthened by the optimal cognitive functions showing the hypothesis applies to CS but not uniformly in AS. This anomaly in the AS is evidenced by the decline of the cognitive functions contributing to abovementioned "linearization" in the graphic representation. There is a direct positive correlation between the results of MARIE and the performance on cognitive tests. Conclusion: The administration of a combination of DRS, FCSRT, and MARIE to patients screened for possibly emerging Alzheimer's could provide a more detailed and specific approach to make a definitive early diagnosis of Alzheimer's. The Alzheimer's patients found it difficult to distinguish between anger and fear.

\section{Keywords}

Recognizing Facial Emotions, Alzheimer Disease, Early Diagnosis

\section{Introduction}

Increasingly, research studies confirm the difficulties of sufferers of Alzheimer's Dementia in the domain of the visual recognition of facial emotions (VRFE) [1]. Throughout the course of the Alzheimer's disease, the problem of not being able to measure the VRFE exists and has been confirmed by many studies [2] [3] [4] [5] [6]. As far as Brosgole is concerned, the recognition of anger is affected from the very beginning at the onset of Alzheimer's [7] [8]. Whereas, Lavenu et al. [4] and Cadieux [9] would refer to fear in the same context. These studies are of an experimental nature, long, and difficult to transpose into a clinical routine. The reasons for the difficulties in adopting these findings for clinical diagnostic purpose are attributable to several factors linked with nature of the tests, namely: 1) a heterogeneous methodology; 2) reliance on verbal responses to designate recognized emotions; 3 ) subjective as opposed to objective evaluation; and 4) selection and matching of control and experimental subjects. As is true for MMSE [10] and other cognitive tests, we would need quantitative measures of VRFE as opposed to subjective and qualitative assessments to better understand the behavioral problems of [AS) Alzheimer's patients [11]. Our impression is that the deficit in VRFE can explain some of the impulsive aggressive reactions of AS. 
This study aims to overcome all of the abovementioned difficulties in objective quantitative measurement of the ability for VRFE by using a simple binary test using the computer mouse without the patient ever having to give any verbal responses. This test, therefore, should be quick, simple, and easy to administer in a clinical setting. In addition, it provides prompt quantitative results that can be easily represented graphically. We measured the VRFE amongst patients who are closer to the onset or at the onset of Alzheimer's and compared those results with the performance of a healthy control group. Next, we examined the criteria used by the subjects in making the decisions in choosing the responses while identifying the emotional expressions.

\section{Method}

The tests have been set up using a software which was developed for this study [Method of Analysis and Research of the Integration of Emotions (MARIE); [12] [13] [14]. It is inspired by a method already used [15] [16] [17] [18]. This test examines the decision making by the subject when shown a photograph of a face which expresses an emotion called “canonical" or “intermediate”. The seven studied canonical emotions [19] are: anger, disgust, fear, happiness, neutral, sadness, and surprise, all of which are expressed by a "blonde woman's face." We have created 9 series of combinations of emotions (SE): anger-fear, anger-sadness, happiness-sadness, neutral-anger, neutral-disgust, neutral-fear, neutral-happiness, neutral-sadness, and neutral-surprise. Each SE was made up of 2 canonical images and 17 intermediate images created by merging two canonical images using computer technology: morphing. The photographs of the "blonde woman" come from "Pictures of Facial Affect" [20].

\section{Participants}

12 voluntary patients in the initial stages of Alzheimer's, following a memory consultation at the Valenciennes' central hospital, were matched with a control group of 12 healthy individuals. All 24 subjects were Caucasians, native to the Northern region of France, French speaking, right-handed and who had given their signed consent. The matching was based on age, sex and educational level. Visual acuity with or without corrective glasses was 20/20. There was no history of previous psychological or neurological disorder or any use of psychotropic medications in the last 6 months. No behavioral disturbances associated with their Major Neurocognitive Disorder had been identified since the onset of their Alzheimer's. The patients would be meeting the criteria of NINCDS-ADRDA [21]. Any symptom like pseudobulbar affect, visual hallucinations associated with Parkinsonian Syndrome that suggested presence of: 1) Lewy body disease [22], 2) Frontal Lobe atrophy [23] [24] (Fronto-temporal Dementia or Pick's Disease) or 3) Basal Cortical degeneration or the Asymmetric Cortical Degenerative Syndrome [25] was a major exclusion criterion for this study.

Performances on the Mini Mental Status Examination (MMSE) [10], the Mat- 
tis Dementia Rating Scale (DRS) [26] [27], the Picture Naming (DO 80) [28], and at the Grober and Buschke's Free and Cued Selective Reminding Test (FCSRT) [29] showed the verbal episodic memory was significantly lower in the Alzheimer's sufferers compared to the control group. Both groups were blinded to the purpose of the study and the functions measured, but all participating researchers were aware of these yet did not intrude or intervene when the subjects were responding to the stimuli. There was no prompting or guidance for the subjects from the research staff administrating the tests (Table 1).

\section{Procedures}

We ran MMSE, DRS, Picture Naming, and FCSRT tests with each of the study's subjects. A sample of 12 healthy individuals were chosen from a population of 204, forming the control group with which the Alzheimer's patients were matched, based on age, sex and level of education.

\section{Setting up of stimuli}

An intermediate emotion does not completely represent either a canonical emotion " $\mathrm{A}$ " or a canonical emotion " $\mathrm{B}$ "- -it is a controlled "combination" which is inversely proportional to the pixels of the two canonical images " $A$ " and " $B$ ". Each series was specified by the increase in the gradation of emotion "B": $0 \%$, $10 \%$, 20\%, 30\%, 35\%, 38\%, 41\%, 44\%, 47\%, 50\%, 53\%, 56\%, 59\%, 62\%, 65\%, $70 \%, 80 \%, 90 \%$, and $100 \%$, (Figure 1).

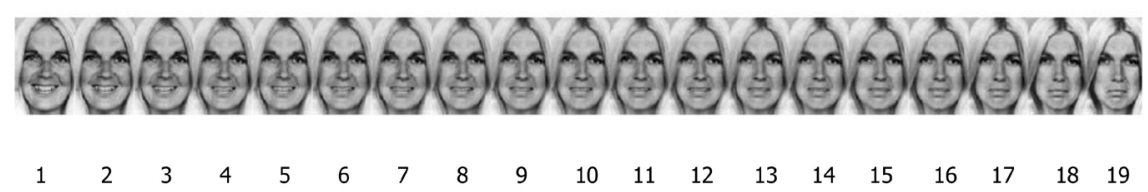

Figure 1. Happiness-sadness series created using the intermediate image merging method, of the canonical images 1 and 19.

Table 1. Subject's characteristics: level of education since age of 4: 1) 12 years of education, 2) 15 years of education, 3) 17 years of education and more; MMSE: Mini Mental Status Examination 144 (mean and standard deviation); Picture Naming/80 (mean and standard deviation); FCSRT (mean and standard deviation).

\begin{tabular}{cccc}
\hline & Control & Alzheimer's & P \\
\hline $\mathbf{n}=$ & group 12 & patients 12 & 1 \\
sex $(\mathrm{M} / \mathrm{F})^{*}$ & $7 / 5$ & $7 / 5$ & 0.3 \\
Age & 66.33 & 68.67 & 0.3 \\
Level of education & $7 / 4 / 1$ & $10 / 1 / 1$ & 0.0005 \\
MMSE $(/ 30)$ & $30( \pm 0.5)$ & $26( \pm 2.4)$ & 0.0005 \\
DRS $(/ 144)$ & $144( \pm 1)$ & $130( \pm 10)$ & 0.0001 \\
Picture Naming $(/ 80)$ & $80( \pm 1)$ & $33( \pm 4)$ & \\
FCSRT: & & & 0.014 \\
Immediate recall & $16( \pm 2)$ & $13( \pm 4)$ & 0.0001 \\
$3^{\circ}$ free recall & $16( \pm 2)$ & $7( \pm 5)$ & 0.0013 \\
$3^{\circ}$ total recall & $16( \pm 2)$ & $11( \pm 6)$ & 0.0013 \\
Total different recall & $16( \pm 2)$ & $11( \pm 6)$ &
\end{tabular}




\section{Procedure}

Each subject was seated in the same quiet room, facing a laptop screen while each of the images $(10 \mathrm{~cm} \times 18 \mathrm{~cm})$ was individually shown. The "stimulus" was shown in the center of the upper half of the screen. On the lower half of the screen: 1) labelled underneath the canonical image, to the left of the stimulus was the name of the canonical emotion " $A$ " (with $5^{\circ}$ angle), 2) labelled underneath the canonical emotion image " $\mathrm{B}$ ", to the right of the stimulus was the name of the canonical emotion " $\mathrm{B}$ " (with a $5^{\circ}$ angle) (Figure 2). The random order of the stimuli shown was the same for each subject $(9$ series $\times 19$ images $=$ 171 stimuli). The canonical images of each series were displayed in the penultimate and ultimate position (First and the last). The 17 intermediate images were displayed in the same random order to every subject.

The subjects undertook a test in which they were obligated to press with their dominant hand on the left or right button of the computer mouse, using the index or the middle finger. The stimulus remained on display until the subject responded. The order in which the series were displayed was as follows: anger-fear, anger-sadness, happiness-sadness, neutral-anger, neutral-disgust, neutral-happiness, neutral-fear, neutral-surprise, and neutral-sadness. The three first combinations are called "bipolar" and the last 6 "unipolar" due to the presence of neutrality in the first set of pictures in each series. A break of one minute was applied after each series which lasted 2 minutes on an average.

The 9 following "measures" have been taken into account: 1) measures numbers $1,2,8$, and 9 corresponded to responses to the stimuli numbers $1,2,18$, and 19 , respectively. 2) measures numbers $3,4,5,6$, and 7 corresponded respectively to the average of the responses to the stimuli numbers 3,4 , and 5; numbers 6,7 , and 8 ; numbers 9,10 , and 11 ; numbers 12,13 , and 14; and numbers 15,16 , and 17 , respectively. The average saturation of emotion B was $0 \%, 10 \%, 28.3 \%, 41 \%$, $50 \%, 59 \%, 71 \%, 90 \%$, and $100 \%$, respectively, for each of the 9 measures.

All of the subjects should have first passed a monitored task. They used the same skill of decision making, a test having to choose between two possible answers, but concerning a series of geometric intermediate images originally from a continuum with square and circular outlines. This initial practice exercise did not involve dealing with recognizing emotions.

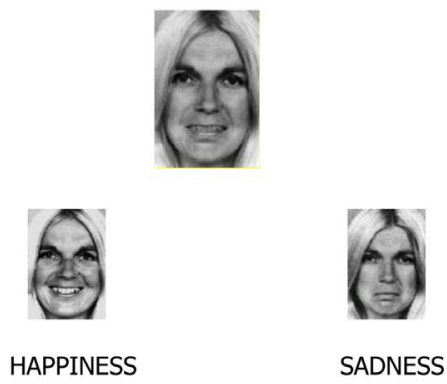

Figure 2. Experimental situation, the subjects must choose the image on the right or the left according to which they identify the central image predominantly representing. 


\section{Statistical Evaluation}

The analysis of the data was carried out by "analyses of variance" (ANOVAs), for which the inter-subjects factor was the group (Alzheimer's or control group), the series (9) and the measures (9) being the intra-subjects factors. The comparison of the qualitative variables was carried out by a Chi-square test. The level of significance was fixed at 0.05 . In all of the analyses, the dependent variable was the percentage of responses "B". We used the software SPSS v. 11.

\section{Results}

We proceeded with four stages. To begin with, (stage 1) we examined the effect of the group, the measure and the difference in series type, the unipolar series in comparison to the bipolar series. One would expect that in the unipolar series, a principally linear interaction will be observed in the measure where the facial expression " $\mathrm{A}$ " is neutrality; on the other hand, for the bipolar series, a clearly sigmoid interaction should appear, which would signify a categorical perception (as various authors have shown since the studies of Etcoff \& Magee, [30]. However, we are aware that some authors consider neutrality to be a real emotional expression (Ekman et Friesen, 1975). Next (stages 2 and 3) we examined the performance of each individual and also as a group with their responses to the stimuli in each series, separately for the three bipolar series and the six unipolar series. Finally, (stage 4) with the aim of selecting the neuropsychological cognitive performances which were best correlated with the inconsistencies in the visual recognition of emotions test, we made a multiple linear regression (with step by step factorial selection), with the sum of the "discordant answers" as the dependent variable and the following factors: MMSE, global score of DRS, subscores of the DRS, subscores of FCSRT. A discordant answer is a different response compared to that of the majority of healthy subjects represented by $(\mathrm{n} / 2)$ +1 .

\section{Stage 1: Effect of series type}

The group $\mathrm{X}$ series $\mathrm{X}$ measure ANOVA revealed a significant main effect of the group $(\mathrm{F}(1,22)=10.2, \mathrm{p}<0.001)$ in favor of the control group (59.36 versus $51.99)$, of the series' type $(\mathrm{F}(1,22)=15.63, \mathrm{p}<0.0007$,$) in favor of the unipolar$ series (59.53 versus 51.82), and of the measure $(\mathrm{F}(8,176)=274.5, \mathrm{p}<0.0001)$. Figure 3 shows the latter effect.

The comparison of measures by orthogonal polynomials show that the linear component is significant $(\mathrm{p}<0.0001)$ and explains $93.7 \%$ of the variance. The rest of the variance is explained by the quadratic $(54 \%$; $p<0.0001)$ and cubic $(19 \% ; p<0.0004)$ components. The curve shows well the appearance of the sigmoid characteristic of categorical perception.

However, these 3 main effects were qualified by several significant interactions. We were particularly interested in the interaction involving the group. The interaction group $\mathrm{X}$ series was not significant $(\mathrm{F}<1)$. The interaction group $\times$ measure $(F(8,176)=8.22, p<0.0001)$ is illustrated by Figure 4. It shows that the 


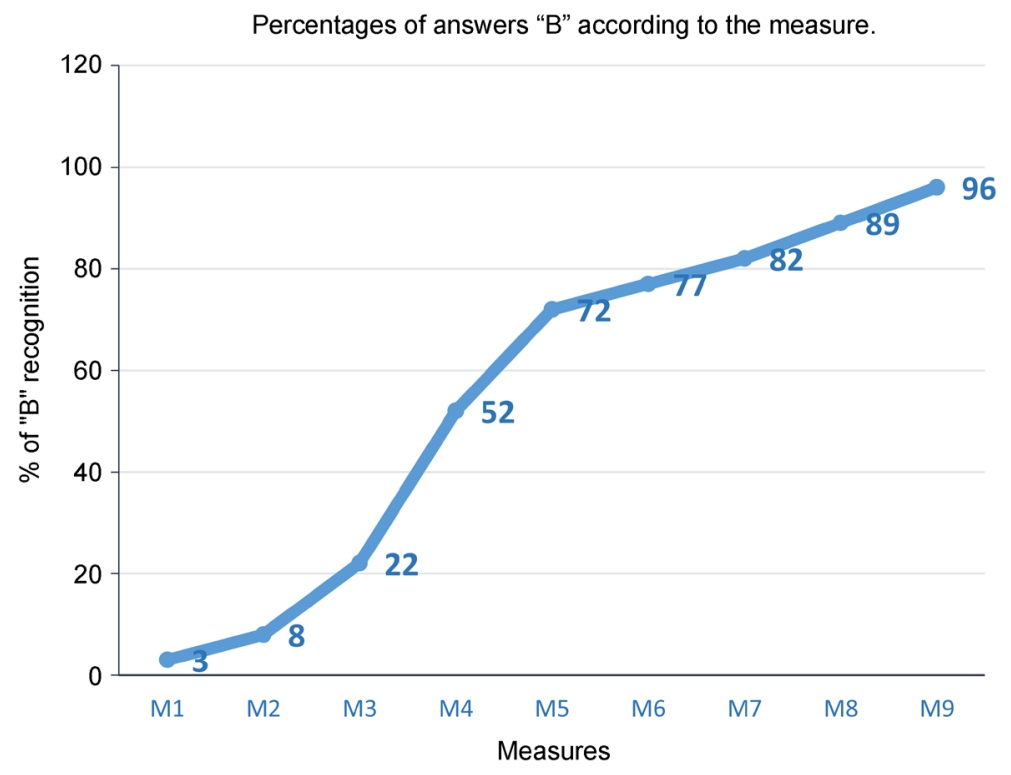

Figure 3. Percentages of answers "B" according to the measure.

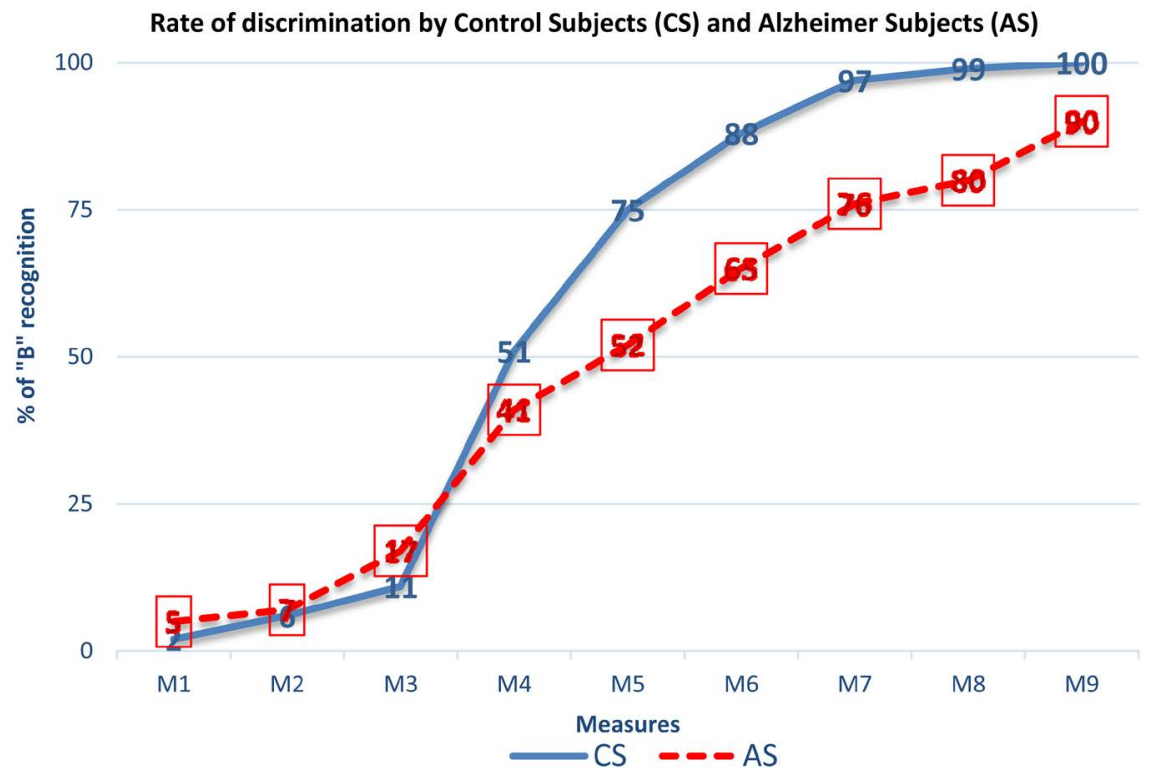

Figure 4. Answer percentage "B" according to group and measure.

sigmoid appearance of the curve is more obvious among the control group than the Alzheimer's patients. In fact, for the control group, the linear component accounted for $89.9 \%$ of the variance $(\mathrm{p}<0.0001), 45 \%$ of the rest was made by the quadratic component $(\mathrm{p}<0.0001)$ and another $45 \%$ by the cubic component ( $p<0.0001)$. Alternatively, among the Alzheimer's patients, the linear component accounted for $96.3 \%$ of the variance $(\mathrm{p}<0.0001)$ and $57 \%$ of the rest was made up by the quadratic component $(\mathrm{p}<0.015)$ and $3 \%$ of the rest by the cubic component (NS). These results would suggest a significant difference in the threshold for emotion identification by the control group and the Alzheimer's patients: 1 ) thus the emotional saturation of $72 \%$ (measure number 7 ) permitted 
an identification of emotion "B" by $95 \%$ of the control group and $77 \%$ of the Alzheimer's patients; and 2) at a 100\% saturation, the Alzheimer's patients progressed to $90 \%$ and the control groups to $100 \%$. The differential average of recognition is $18 \%$ for the measures 5 to 9 . An emotion was recognized by an equal number of Alzheimer's patients as control members as long as the images had $18 \%$ more of "B" pixels in addition to the images that were shown to the control group. As for the interaction series $\mathrm{X}$ measure $(\mathrm{F}(8,176)=6.65 ; \mathrm{p}<0.0001)$, it revealed a more marked sigmoid appearance for the unipolar series than for the bipolar series, contrary to our expectations.

Again, all of the effects were adjusted by the triple interaction $(\mathrm{F}(8,176)=$ $5.43, \mathrm{p}<0.0004)$. We analyzed this interaction making an ANOVA group $\times$ measure for each separate series. For the unipolar series (Figure 5, left side), as well as the main effects, the interaction group $\times$ measure was significant $(\mathrm{F}(8$, $176)=248, \mathrm{p}<0.0001)$. Among the control group, the curve was clearly sigmoid: the linear component only made up $83.1 \%$ of the variance ( $p<0.0001$ ), $60 \%$ of the rest being explained by the quadratic component and $8 \%$ by the cubic component $(p<0.0002)$. On the other hand, among the Alzheimer's patients, the linear component accounted for $94.5 \%$ of the variance ( $\mathrm{p}<0.0001$ ), $53 \%$ of the rest being the quadratic component $(\mathrm{p}<0.008)$ and $9 \%$ the cubic component (NS). In the following analyses, we shall mainly focus on the effect of the series, as the effects of the group and measure have already been explored in the preceding analyses.

\section{Stage 2: analysis of the bipolar series}

In the ANOVA group (2) $\times$ series $(3) \times$ measure (9), the only significant main effect was of the measure $(\mathrm{F}(8,176)=132.8, \mathrm{p}<0.0001)$, suggesting an important linear component ( $97 \%$ of the variance, $\mathrm{p}<0.0001$ ).

However, several interactions were significant. Confirming the previous analysis (Figure 5, right side), the interaction group $\times$ measure was significant $(\mathrm{F}(8,176)=8.51, \mathrm{p}<0.0001)$ : the curve is sigmoid for the control group and linear for the Alzheimer's patients. The series did not interact with the measure $(\mathrm{F}(16,352)=1.28, \mathrm{NS})$, but did with the group $(\mathrm{F}(2,44)=6.74, \mathrm{p}<0.004)$. This
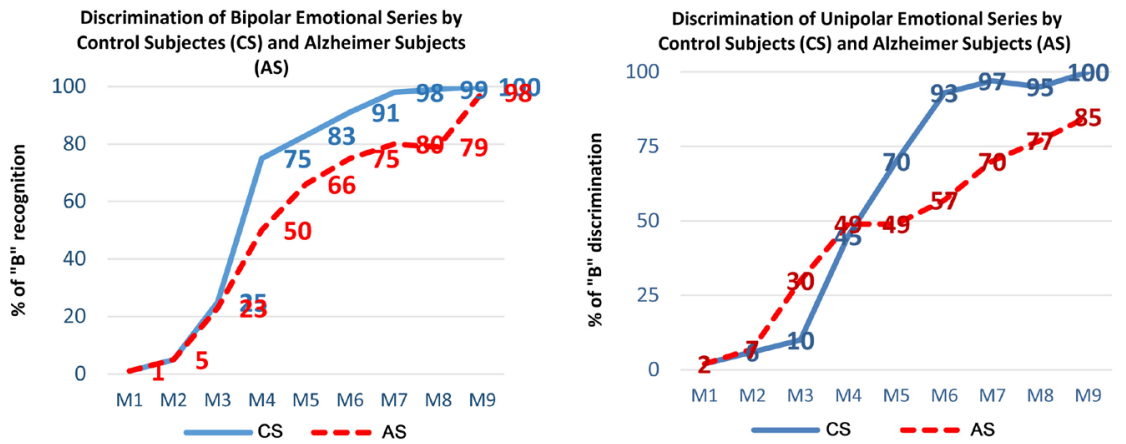

Figure 5. On the left a comparison between the control group and the Alzheimer's patients, for the recognition of " $\mathrm{B}$ " for all of the bipolar series; and, on the right, for all of the unipolar series. 
interaction (Figure 6) shows the figures for the answer " $\mathrm{B}$ ": 1 ) for the anger-fear series $(p<0.002)$, the rate is higher for the control group than the Alzheimer's patients, without any effect for the two other series; 2 ) the rate for control group is higher for the anger-fear and the anger-sadness series (which do not differ from each other) than for the happiness-sadness series $(\mathrm{p}<0.016)$; and 3$)$ the rate for the Alzheimer's patients is higher for the happiness-sadness and anger-sadness series (which do not differ from each other) than for the anger-fear series.

In short, the interaction between the three factors was significant $(\mathrm{F}(16,352)=$ 4.4, $\mathrm{p}<0.0002)$. This is represented in Figure 7. In order to analyze it, we proceeded to an ANOVA group $\times$ measure for each separate series. For the anger-fear series, the main effect of the group was significant $(\mathrm{F}(1,22)=18.22, \mathrm{p}<$ 0.0003) in favor of the control group, in the same way as the main effect of the measure $(F(8,176)=43.48, p<0.0001)$. However, the interaction was not significant $(\mathrm{F}(8,176)=1.86, \mathrm{NS})$. For the two joined groups, the curve showed a strong linear component ( $98 \%$ of the variance explained, $\mathrm{p}<0.0001)$. There was,

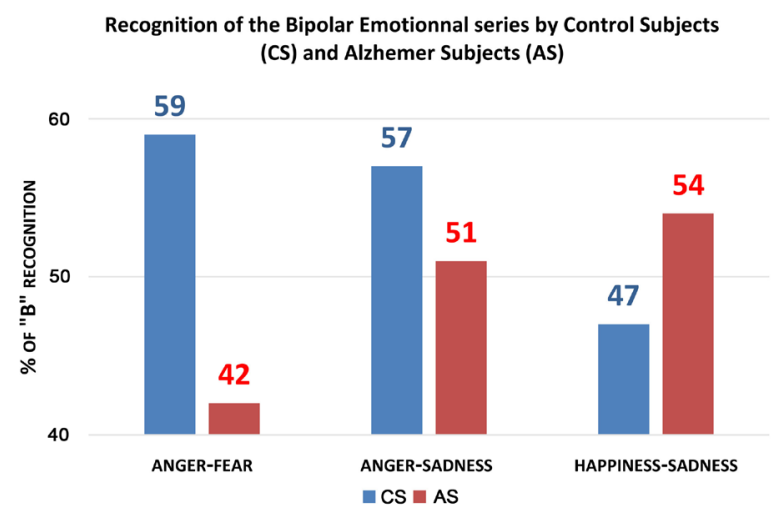

Figure 6. Comparison between Alzheimer's patients and control group, for the bipolar series.
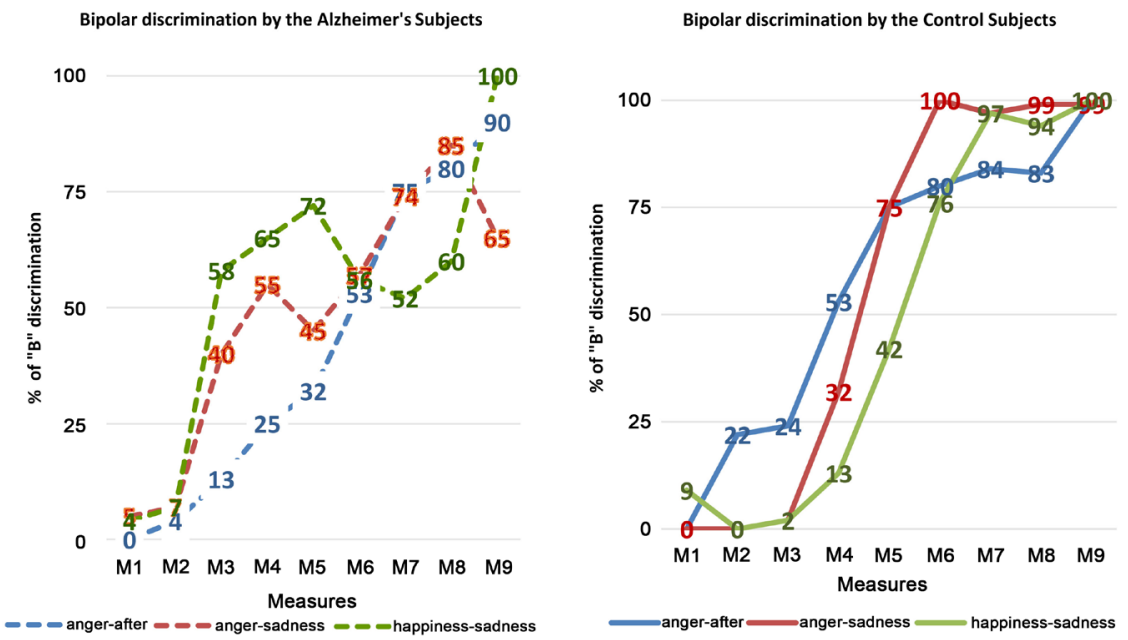

Figure 7. Comparison between the Alzheimer's patients and the control group, for the recognition of "B" in the bipolar series. 
however, a significant interaction between the groups and the measure. For the control group, the sigmoid appearance of the curve was clear: the linear component explained $87 \%$ of the variance ( $\mathrm{p}<0.0001$ ), $22 \%$ of the rest was the quadratic component $(\mathrm{p}<0.0001)$ and $53 \%$ was the cubic component $(\mathrm{p}<0.0001)$. Alternatively, for the Alzheimer's patients, the curve was purely linear $(79.4 \%$ of the variance, $\mathrm{p}<0.0001$ ), the two other components not being significant. Finally, for the series happiness-sadness, there was not a major effect from the group but there was from the measure $(\mathrm{F}(8,176)=42.9, \mathrm{p}<0.0001)$. However, there was a significant interaction between the group and the measure $(\mathrm{F}(8,176)=$ $8.5, \mathrm{p}<0.0001)$. For the control group, the linear component made up $89 \%$ of the variance $(\mathrm{p}<0.0001), 6 \%$ of the rest of the variance was accounted for by the quadratic component and $82 \%$ by the cubic component $(\mathrm{p}<0.0003)$.

A more detailed observation allows us to say that the discrimination of "B" by AS for 3 SEs called bipolar. B is fear in the SE anger-fear, sadness in the SE anger-sadness and sadness in the SE happiness-sadness. The appearance of curves is diagonal line for the first two and stair-step pattern for the third, suggesting that the answer was arrived at random by fluke or $50 \%$ chance like flipping of the coin in the happiness-sadness series. In the anger-sadness series the AS quickly identifies sadness beginning at M1. It exceeds 50\% at M4. The Alzheimer subjects do not perceive sadness in M1, M2 and M3. The measures from M4 to M9 oscillate in a band between $45 \%$ and $80 \%$. The canonical emotion sadness is identified as anger by $35 \%$ of Alzheimer subjects. The latter seem incapable of differentiating anger from sadness. The control subjects distinguish anger from sadness quite well. The emotional series anger-sadness would be very sensitive to cognitive disorders caused by Alzheimer's disease. In the happiness-sadness series we have to go beyond M5 to perceive as much happiness as sadness in the control subjects. In contrast for AS from M3 the perception of sadness is $58 \%$. Then, from M3 to M8 the recognition of sadness oscillates between $50 \%$ and $75 \%$.

Alzheimer subjects have difficulty distinguishing between happiness and sadness. This emotional happiness-sadness series is strongly impacted by the cognitive disorders induced by Alzheimer's Disease. A major worsening of Alzheimer's disease would impose a total inability to distinguish between happiness and sadness. Then, the patients would respond randomly and we would have an almost horizontal curve which would oscillate around 50\% for each measurement of M. In other words, the band $50 \%-75 \%$ is a zone of confusion where the distinguishing of happiness from sadness is difficult. However, the confusion is resolved by preferring sadness. The AS is unable to differentiate happiness from sadness. When AS is unable to recognize happiness AS confuses it with sadness. This explains how ASs mistake happiness for sadness. A first hypothesis would be that there is an organic inability to distinguish happiness from sadness due to neurodegenerative lesions in the neural circuits operating in the recognition of emotional expressions. A second hypothesis would be that the AS experience a sad mood with pervasive sadness which AS is not able to express but which 
would be unmasked by presentation of the bipolar series happiness-sadness. This hypothesis would be confirmed or invalidated by implementing effective, robust treatment with antidepressant medications with repeat testing after six weeks. Perhaps even much sooner than in six weeks when treated with esketamine which is more robust and expeditious. The bipolar happiness-sadness series would also be one of the two tools embedded in MARIE to screen for early Alzheimer's disease.

The anger-fear series shows that it is graphically represented in a diagonal pattern. We go from a categorical perception to a one-dimensional perception. Alzheimer subjects perceive 50\% of fear in M6 while the healthy subject perceives them in M4. Therefore, this study clearly shows Alzheimer Subjects confuse anger and fear. They see anger where there is fear. A first hypothesis would be: This is a result of an organic/neuronal degeneration in the emotion recognition circuits in ASs. The second hypothesis would be a pervasive emotional state where the Alzheimer subjects are in a subclinical angry mood. This confusion between anger and fear can explain aggressiveness towards other by projecting anger. The Alzheimer Subject wrongly perceives a person who expresses fear as angry. He interprets fear as anger. He attacks the person who is expressing fear because AS considers himself to be in danger.

The anger-fear bipolar series would also be a good tool embedded in MARIE for early detection of Alzheimer's disease.

In each emotional series which combines anger with another emotion, it is obvious the Alzheimer subject preferentially perceives anger.

\section{Stage 3: analysis of the unipolar series.}

In the ANOVA group (2) $\times$ measure (9), the three main effects were significant (for the first two, see the initial analysis, Figure 5, left graph). The number of those who answered "B" was higher among the control group than the Alzheimer's patients $(\mathrm{F}(8,176)=247.6, \mathrm{p}<0.0001)$, and varied according to the series $(\mathrm{F}(5,110)-6.10, \mathrm{p}<0.0006)$. These three effects were yet to be qualified concerning certain interactions.

The interaction group $\times$ series was not significant $(\mathrm{F}<1, \mathrm{NS})$. The interaction group $\times$ measure was significant $(F(8,176)=5.21, \mathrm{p}<0.0025)$ : as we saw in the initial analysis, the curve is sigmoid for the control group, but mostly linear for the Alzheimer's patients. Next, the series $\times$ measure interaction was equally significant $(\mathrm{F}(40,880)=2.04, \mathrm{p}<0.04)$ but, as they concern mixed groups we will not analyze them very much. Finally, the interaction between the three factors was not significant $(\mathrm{F}(40,880)=1.2$, NS).

\section{Stage 4: multiple regression}

The correlations studied by the test Bravais Pearson between the sum of discordant answers and neuropsychological performance showed a significant correlation with: 1$)$ the MMSE $(r=-0.851, p=0.0001), 2)$ the aggregate scores of DRS $(\mathrm{r}=-0.93, \mathrm{p}=0.0001), 3)$ sub scores of DRS : a) name $(\mathrm{r}=-0.82, \mathrm{p}=$ $0.001), \mathrm{b})$ fluence animals $(\mathrm{r}=-0.82, \mathrm{p}=0,001, \mathrm{c})$ Fluence $\mathrm{P}(\mathrm{r}=-0.579, \mathrm{p}=$ 0.049), 4) sub scores of FCSRT: a) free recall $1(r=-0.773, p=0.003)$, b) free 
recall $3(\mathrm{r}=-0.757, \mathrm{P}=0.004), \mathrm{c})$ delayed free recall $(\mathrm{r}=-0.811, \mathrm{p}=0.001), \mathrm{d})$ reminder total deferred $(r=-0.717, p=0.009)$. For selecting the best parameters indicative of neuropsychological disturbances that correlated with the poor performance on the test of visual recognition of emotions (MARIE), we conducted a multiple linear regression with selection factor step by step with the sum of discordant answers as dependent variable and the following factors: MMSE, score Overall DRS, scores of DRS, sub-scores of FCSRT. The following factors were selected: overall score DRS $\left(\mathrm{r}^{2}=0.79, \mathrm{p}=0.001\right)$ and delayed free recall as reflected by the score of FCSRT $\left(r^{2}=0.1, p=0.013\right)$. In this manner, the trouble with the recognition of emotions was linked to the severity of the dementia syndrome measured by the deficit in the episodic verbal memory and in overall decline in intellectual efficiency ("mental abilities").

\section{Discussion}

Our results confirm that visual recognition of facial emotion is already altered during the early stages of Alzheimer's after its onset, which is in agreement with some authors [3] and in disagreement with others concerning this phase of the illness [31] [32]. We note the existence of increased threshold: 1) the Alzheimer's patients would be markedly less sensitive to the visual cues of facial emotions, meaning they have a higher threshold for recognizing them, 2) the facial expression of emotions of any subject must be "exaggerated" so the Alzheimer's patients are able to identify it in the same manner as the control group. The similar threshold for all of the series allows discussion of the way that one single region of the brain is affected. The involvement of the prefrontal cortex and its declined function is called to mind as likely the most significant factor.

The very poor recognition of fear in the series anger-fear, confirms on one hand the hypothesis of dysfunction of the amygdala in the very first phases of Alzheimer's and on the other hand, it's involvement in the recognition of anger. In this eventuality, the association of a deficit: 1) with the recognition of fear in the series anger-fear, 2) with the global score DRS, and 3) with the delayed free recall of the FCSRT score, are some of the characteristics of Alzheimer's. Cadieux and Greve [9], confirm the existence of emotion recognition problems in Alzheimer's which would be secondary to the histopathological damage present in the amygdala-hippocampus complex [9] [33] [34]. The level of recognition of fear in the anger-fear series is worse than in the rest of the series'. This is linked to confusion when distinguishing between anger and fear. This confusion makes ASs choose anger. In other words, the expression of fear on the face of someone that a demented person is talking to could be interpreted as anger by AS. It would be a possible explanation for some acts of aggressiveness demonstrated by Alzheimer's patients, all the more during the more advanced phases of the illness. These characteristics and the sensitiveness of this series makes it unique. Therefore, anger-fear series could be used as an aid at pre-screening clinics designed for Alzheimer's diagnosis. The worsening of Alzheimer's could be linked to a decline in the ability to recognize a large number of affected emotions. These 
findings reinforce the results of Spoletini [35] and Teng [36].

In the graphic representation, the appearance of the curve is sigmoid for the control group and linear for the Alzheimer's patients with a cognitive distortion occurring in this domain. A sigmoid curve is suggestive of an abrupt transition in the recognition of emotion expression from one state to another. This can be explained as follows. The control subject is certain of his choices. There is no doubt in the recognition of emotion within and beyond a certain limit. In this case, the decision making is categorical. The control subject identifies the facial emotions of others correctly and without hesitation. This type of near preciseness in recognizing facial expressions of emotions results is a successful social adjustment.

The linear curves are not easy to interpret at an individual level, all the more so in the case of a binary option. The amount of time taken to make a decision (later publication) in AS shows the hesitation when faced with the central images of the series. The answers seem proportional for the percentage of " $\mathrm{B}$ " pixels in each measure. We formulate the hypothesis of the inability to link each intermediate image to an "archetypal image" of each of the emotions that are stored in the memory. For our purpose we may call it a "reference image". We also allow for the possibility that in AS the archetypal or reference image is fading gradually like the polaroid camera pictures. The conviction of being sure of having made the right choice is only gradually acquired as a consequence of the unresolved conscious anxiety of not correctly identifying the facial emotion of the person the subjects are engaging with. This type of anxiety could explain the reluctance of Alzheimer's sufferers to engage in social and familial relations, which then consequently leads to a withdrawal from society and difficulty in being part of emotionally intense situations, such as weddings, birthdays, etc. even among familiar faces of the members of the family or close friends.

The emotional deficit is significant (control group: 59.36; Alzheimer's patients: 51.99) despite a slight reduction of the DRS (144 $( \pm 1)$ versus $130( \pm 10)$; p $<0.0005)$. However, it is hard to claim for certain that the recognition system is controlled purely by the cognitive system (cerebral cortex) which gradually loses this faculty of VRFE, or if there are actually two distinct systems which are simultaneously vulnerable to the deleterious effect of neurofibrillary degeneration and increased intracellular accumulation of the beta-amyloid protein in the neurons. A longitudinal study of a mature population, looking at the measure of cognition and emotion recognition could answer this question in the future. In other words, consistently finding significant decline in VRFE in the early stages of AS associated with no remarkable evidence of cognitive decline or only mild cognitive decline will implicate subcortical involvement (amygdala-hippocampus complex) as the most likely culprit in initial stages of Alzheimer's.

\section{Conclusions}

This study underlined: 1) a problem with the visual recognition of facial emo- 
tion, 2) and the existence of limitations in the visual recognition of emotions by Alzheimer's sufferers. The Alzheimer's patients found it difficult to distinguish between anger and fear. This difficulty could be related to a dysfunction of the amygdala-hippocampus complex. The anger-fear series seems to be particularly sensitive during the onset of Alzheimer's and could be used in the early diagnosis of this disease.

The categorical processing of emotions seems to be strengthened by and linked to optimal cognitive functions. Conversely, the decline in the (higher Cortical) cognitive functions contributes to its "linearization". In this circumstance, the Alzheimer's patients would have doubts about their interpretation of facial emotion. This linearization of a categorical recognition aspect could be extrapolated to the recognition and naming of faces, objects, discerning nuances in language, etc. due to disappearance of the "archetypal" or "reference" information akin to the fading of the polaroid camera pictures over time. Initially the facial expressions require more attention to be recognized. Then eventually it is impossible for AS to recognize them. As a result, there is: 1) hesitation, 2) anxious perplexity, 3) a lengthening in decision making time which leads to the demented person becoming socially isolated in order to avoid making the wrong choices during social interactions. The worsening of the Alzheimer's leads to a hesitant or equivocal response to hazard with $50 \%$ answer " $A$ " and $50 \%$ answered " $B$ " for each choice. Thus, the linearization moving towards a horizontalization of the graph (later publication).

The limitation of the study is that it suffers from conclusions being drawn from findings in a small cross-section of only one homogeneous population. However, it does confirm and clarify findings published in other studies and discoveries revealed therein. The simplicity of the tool, MARIE, does not take away its accuracy, its validity, and reliability probably due to having had a better understanding of the Alzheimer's patients gained from our multiple studies using this very tool.

The two measures, DRS and FCSRT, improve the discrimination of each of the two groups. The combination of these 3 measures DRS, FCSRT including MARIE could allow a more detailed and specific approach in the early diagnosis of Alzheimer's. Our humble assertion is that our tool, MARIE, can be legitimately considered to supplement these other neuropsychological tools in the early diagnosis, or for even clinching the diagnosis of Alzheimer's Dementia when ambiguities in diagnosis exist. Furthermore, when more robust and effective treatments for arresting or reversing the devastating relentless cognitive and functional decline in this disease become available, MARIE may play a major role in confirming early diagnosis to help confidently initiate such definitive specific effective treatment and preventive measures. We cannot overemphasize the role of the tools like MARIE in preventing and arresting the cognitive decline in Alzheimer's Dementia when effective treatment modalities with specific indications in cognitive domain become available.

These results allow the extrapolation of using the principle "must select one of 
two answers in the perceptive continuum" of our tool to enhance its versatility: MARIE has the potential for use in quantifying accurate perception or discrimination in the area of visual recognition of human faces or images, voice recognition, and also of other speech sounds in auditory domains.

The insight provided by this study will increase empathy for the Alzheimer's victim in the clinical and caregiver support staff to help in the behavioral management of AS, as well as in relating to AS at various stages of this disease. The study will also invite new respect for the socially competent people who are savvy at VRFE by recognizing how they can be more empathic in their social interactions especially if they choose a career involving a need for more astuteness of this ability for VRFE.

\section{Acknowledgements}

This clinical research and this clinical study were supported by the grant 1998/ 1954 of the Programme Hospitalier de Recherche Clinique of the French government. Thanks are due to Paul Ekman who gave permission to use photographs from "Unmasking the Faces" (Ekman \& Friesen, 1975) and to Olivier Lecherf who designed the computer program for processing and displaying the pictures. This study was made possible thanks to the Centre d'Investigation Clinique (CIC-CHU/INSERM, Lille) and the Laboratoire de Neurosciences Fonctionnelles \& Pathologies CNRS UMR 8160 CHRU Lille. The authors thank Miss Latitiae Dusart for her collaboration and advice in neuropsychology. The authors also thank Jay Vinekar, MBA, of Houston, Texas for editorial help and help with translation. Finally, the authors thank Madame Marie-Pierre Bongiovanni, the Executive Director of the Centre Hospitalier Sambre Avesnois, for her help and encouragement.

\section{Conflicts of Interest}

The authors declare no conflicts of interest regarding the publication of this paper.

\section{References}

[1] Hargrave, R., Maddock, R.J. and Stone, V. (2002) Impaired Recognition of Facial Expressions of Emotion in Alzheimer's Disease. The Journal of Neuropsychiatry and Clinical Neurosciences, 14, 64-71. https://doi.org/10.1176/jnp.14.1.64

[2] Shimokawa, A., Yatomi, N., Anamizu, S., Torii, S., Isono, H. and Sugai, Y. (2003) Recognition of Facial Expressions and Emotional Situations in Patients with Dementia of the Alzheimer and Vascular Types. Dementia and Geriatric Cognitive Disorders, 15, 163-168. https://doi.org/10.1159/000068479

[3] Burnham, H. and Hogervorst, E. (2004) Recognition of Facial Expressions of Emotion by Patients with Dementia of the Alzheimer Type. Dementia and Geriatric Cognitive Disorders, 18, 75-79. https://doi.org/10.1159/000077813

[4] Lavenu, I. and Pasquier, F. (2005) Perception of Emotion on Faces in Frontotemporal Dementia and Alzheimer's Disease: A Longitudinal Study. Dementia and Geriatric Cognitive Disorders, 19, 3741. https://doi.org/10.1159/000080969 
[5] Kolher, C.G., Anselmo-Gallagher, G., Bilker, W., Karlawish, J., Gur, R. and Clark, C.M. (2005) Emotion-Discrimination Deficits in Mild Alzheimer Disease. The American Journal of Geriatric Psychiatry, 13, 11. https://doi.org/10.1097/00019442-200511000-00002

[6] Bucks, R.S. and Radford, S.A. (2004) Emotion Processing in Alzheimer's Disease. Aging et Mental Health, 8, 222-232. https://doi.org/10.1080/13607860410001669750

[7] Brosgole, L., Kurucz, J., Plahovinsak, T.J., Sprotte, C. and Haveliwala, Y.A. (1983) Facial- and Postural-Affect Recognition in Senile Elderly Persons. International Journal of Neuroscience, 22, 37-45. https://doi.org/10.3109/00207459308987383

[8] Brosgole, L. and Weisman, J. (1995) Mood Recognition across the Ages. International Journal of Neuroscience, 82, 169-189. https://doi.org/10.3109/00207459508999800

[9] Cadieux, N.L. and Greve, K.W. (1997) Emotion Processing in Alzheimer's Disease. Journal of the International Neurological Society, 3, 411-419. https://doi.org/10.1017/S1355617797004116

[10] Folstein, M.F., Folstein, S.E. and McHugh, P.R. (1975) Mini-Mental State: A Practical Method for Grading the Cognitive State of Patients for the Clinician. Journal of Psychiatry Research, 12, 189-198. https://doi.org/10.1016/0022-3956(75)90026-6

[11] Shimokawa, A., Yatomi, N., Anamizu, S., Torii, S., Isono, H., Sugai, Y. and Kohno, M. (2001) Influence of Deteriorating Ability of Emotional Comprehension on Interpersonal Behavior in Alzheimer-Type Dementia. Brain and Cognition, 47, 423 433. https://doi.org/10.1006/brcg.2001.1318

[12] Granato, P. and Bruyer, R. (1996) Étude objective de la perception du sourire et de la tristesse par la méthode d'analyse de recherche de l'intégration des émotions MARIE. Annales Médico-Psychologiques, 154, 1-9.

[13] Bruyer, R. and Granato, P. (1999) Categorical Effects in the Perception of Facial Expressions: MARIE. A Simple and Discriminating Clinical Tool. European Review of Applied Psychology, 49, 3-10.

[14] Granato, P. and Bruyer, R. (2002) Measurement of Facially Expressed Emotions by a Computerized Study: Method of Study and Analysis of Integration of Emotions (MARIE). European Psychiatry, 17, 339-348. https://doi.org/10.1016/S0924-9338(02)00684-3

[15] Calder, A.J., Young, A.W., Perrett, D.I., Etcoff, M.L. and Rowland, D. (1996) Categorical Perception of Morphed Facial Expressions. Visual Cognition, 3, 81-117. https://doi.org/10.1080/713756735

[16] Calder, A.J., Young, A.W., Rowland, D., Perrett, D.I., Hodges, J.R. and Etcoff, N.L. (1996) Facial Emotion Recognition after Bilateral Amygdala Damage: Differentially Severe Impairment of Fear. Cognitive Neuropsychology, 13, 699-745. https://doi.org/10.1080/026432996381890

[17] De Gelder, B., Teunisse, J.P. and Benson, P.J. (1997) Categorical Perception of Facial Expressions: Categories and Their Internal Structure. Cognition and Emotion, 11, 1-23. https://doi.org/10.1080/026999397380005

[18] Young, A.W., Rowland, D., Calder, A.J., Etcoff, N.L., Seth, A. and Perrett, D.I. (1997) Facial Expression Mega Mix: Tests of Dimensional and Category Accounts of Emotion Recognition. Cognition, 63, 271-313. https://doi.org/10.1016/S0010-0277(97)00003-6

[19] Ekman, P. and Friesen, W.V. (1975) Unmasking the Face. Prentice Hall, Englewood Cliffs.

[20] Ekman, P. and Friesen, W.V. (1976) Pictures of Facial Affect. Consulting Psycholo- 
gists Press, Palo Alto.

[21] Mc Khann, G., Drachman, D., Folstein, M., Katzman, R., Price, D. and Stadlan, E.M. (1984) Clinical Diagnosis of Alzheimer's Disease: Report of the NINCDSADRDA Work Group under the Auspices of Department of Health and Human Services Task Force on Alzheimer's Disease. Neurology, 34, 939-944. https://doi.org/10.1212/WNL.34.7.939

[22] MC Keith, I., Mintzer, J., Aarsland, D., Burn, D., Chiu, H., Cohen-Mansfield, J., Dickson, D., Dubois, B., Duda, J.E., Feldman, H., Gauthier, S., Halliday, G., Lawlor, B., Lippa, C., Lopez, O.L., Carlos Machado, J., O’Brien, J., Playfer, J. and Reid, W. (2004) International Psychogeriatric. Association Expert Meeting on DLB. Dementia with Lewy Bodies. The Lancet Neurology, 3, 19-28.

https://doi.org/10.1016/S1474-4422(03)00619-7

[23] Mesulam, M.M. (2001) Primary Progressive Aphasia. Annals of Neurology, 49, $425-$ 432. https://doi.org/10.1002/ana.91

[24] Snowden, J.S. and Neary, D. (1993) Progressive Language Dysfunction and Lobar Atrophy. Dementia, 4, 226-231. https://doi.org/10.1159/000107327

[25] Caselli, R.J., Jack, C.R., Petersen, R.C., Wahner, H.W. and Yanagihara, T. (1992) Asymmetric Cortical Degenerative Syndromes: Clinical and Radiologic Correlations. Neurology, 42, 1462-1468. https://doi.org/10.1212/WNL.42.8.1462

[26] Mattis, S. (1976) Mental Status Examination for Organic Mental Syndrome in Elderly Patients. Grune et Stratton, New York.

[27] Lucas, J.A., lvnik, R.J., Ge, S., Bohac, D.L., Tangalos, E.G. and Kokmen, E. (1998) Normative Data for the Mattis Dementia Rating Scale. Journal of Clinical and Experimental Neuropsychology, 20, 536-547. https://doi.org/10.1076/jcen.20.4.536.1469

[28] Deloche, G., Hannequin, D., Dordain, M., Perrier, D., Pichard, B., Quint, S., MetzLutz, M.N., Kremin, H. and Cardebat, D. (1996) Picture Confrontation Oral Naming: Performance Differences between Aphasics and Normals. Brain and Language, 53, 105-120. https://doi.org/10.1006/brln.1996.0039

[29] Buschke (1984) Cued Recall in Amnesia. Journal of Clinical Neuropsychology, 6, 433-440. https://doi.org/10.1080/01688638408401233

[30] Etcoff, N.L. and Magee, J.J. (1992) Categorical Perception of Facial Expressions. Cognition, 44, 222-240. https://doi.org/10.1016/0010-0277(92)90002-Y

[31] Labar, K.S., Mesulam, M., Gitelman, D.R. and Weintraub, S. (2000) Emotional Curiosity: Modulation of Visuospatial Attention by Arousal Is Preserved in Aging and Early-Stage Alzheimer's Disease. Neuropsychologia, 38, 1734-1740. https://doi.org/10.1016/S0028-3932(00)00077-4

[32] Labar, K.S., Torpey, D.C., Cook, C.A., Johnson, S.R., Warren, L.H., Burke, J.R. and Welsh-Bohmer, K.A. (2005) Emotional Enhancement of Perceptual Priming Is Preserved in Aging and Early Stage Alzheimer's Disease. Neuropsychologia, 43, 1824 1837. https://doi.org/10.1016/j.neuropsychologia.2005.01.018

[33] Braak, H. and Braak, E. (1991) Neuropathological Staging of Alzheimer-Related Changes. Acta Neuropathologica, 82, 239-259. https://doi.org/10.1007/BF00308809

[34] Van Hoesen, G.W. (1997) Ventromedial Temporal Lobe Anatomy, with Comments on Alzheimer's Disease and Temporal Injury. Journal of Neuropsychiatry, 9, 331341. https://doi.org/10.1176/jnp.9.3.331

[35] Spoletini, I., Marra, C., Di Iulio, F., Gianni, W., Sancesario, G., Giubilei, F., Trequattrini, A., Bria, P., Caltagirone, C. and Spalletta, G. (2008) Facial Emotion Recognition Deficit in Amnestic Mild Cognitive Impairment and Alzheimer Disease. Ameri- 
can Journal of Geriatric Psychiatry, 16, 389-398.

https://doi.org/10.1097/JGP.0b013e318165dbce

[36] Teng, E., Lu, P.H. and Cummings, J.L. (2007) Deficits in Facial Emotion Processing in Mild Cognitive Impairment. 2007. Dementia and Geriatric Cognitive Disorders, 23, 271-279. https://doi.org/10.1159/000100829 